ÉGYPTE

monde arabe

\section{Égypte/Monde arabe}

7| 1991

Perceptions de la centralité de l'Égypte 1

\title{
Youssef Idris : Quelques souvenirs
}

Ibdâ' n 9, septembre 1991

\section{Karam Mutâwa'}

\section{(2) OpenEdition}

Journals

Édition électronique

URL : https://journals.openedition.org/ema/1193

DOI : 10.4000/ema.1193

ISSN : 2090-7273

Éditeur

CEDEJ - Centre d'études et de documentation économiques juridiques et sociales

Édition imprimée

Date de publication : 30 septembre 1991

Pagination : 183-188

ISSN : 1110-5097

Référence électronique

Karam Mutâwa', «Youssef Idris : Quelques souvenirs », Égypte/Monde arabe [En ligne], 7| 1991, mis en ligne le 08 juillet 2008, consulté le 07 juillet 2022. URL : http://journals.openedition.org/ema/1193 ; DOI : https://doi.org/10.4000/ema.1193

Ce document a été généré automatiquement le 7 juillet 2022

Tous droits réservés 


\title{
Youssef Idris : Quelques souvenirs
}

\author{
Ibdâ' n 9, septembre 1991
}

\author{
Karam Mutâwa'
}

\section{NOTE DE L'ÉDITEUR}

Karam Mutâwa' est le premier metteur en scène d'Al-Farâfir (NdT).

Janvier 1964...

2 Je venais de rentrer d'Italie après avoir achevé mes études à l'Académie nationale de Rome. La culture et l'art dramatique en Egypte étaient alors au cœur de mes préoccupations - mon départ, à la fin de l'année 1958, ne m'en avait pas éloigné, non plus que les valeurs qui m'imprégnaient et m'avaient accompagné tout au long de mes études en Italie : une foule de grands noms dont je me représentais la valeur tout en entamant mon itinéraire, mon dialogue sérieux avec le théâtre digne de ce nom; des noms du monde littéraire, auteurs dramatiques ou critiques, metteurs en scène... J'y reconnaissais et y reconnais toujours des maîtres, des symboles, des pionniers. J'ai eu l'honneur de travailler à leurs côtés... dans leur ombre... cherchant un espace où je pourrais affirmer ma spécificité.

3 C'est alors que je le rencontrai. Il m'apportait une pièce en trois actes, Al-farâfîr... Au début, le titre m'a décontenancé... Je commençai par lui demander à quoi il faisait référence dans le patrimoine... La réponse ne fut pas claire. Finalement, il me dit : «Je vous ai choisi pour mettre en scène cette pièce que mes maîtres ont rejetée... soit qu'ils ne l'aient pas comprise, soit qu'elle ne les ait pas enthousiasmés... On tient à votre sujet des propos laissant entendre que vous êtes habité par quelque folie créatrice, c'est cette folie que j'attends de vous. »

4 Je lui ai demandé une journée de répit... J'ai lu la pièce une première fois, une deuxième, puis une troisième fois... et lui ai dit : «Je suis emballé par les deux premiers actes ; quant au troisième, je le sens étranger à la texture générale de la pièce, qui est profondément ancrée dans la cause dont elle traite et l'expose de façon tangible, alors 
que ce troisième acte plane dans les hauteurs de la métaphysique. » Il a répondu : "Voici la première des manifestations de folie à laquelle je m'attendais... »

Je me suis engagé à mettre en scène les deux premiers actes pour une représentation au Théâtre national (le troisième acte, que nous avons écarté, constituera plus tard le thème d'une nouvelle pièce intitulée Al-mahzala al-ardhiyya ("La Comédie terrestre »). Alors commença entre nous deux une série de disputes plutôt plaisantes - tel est mon point de vue d'aujourd'hui, mais à l'époque, ces désaccords étaient vifs, c'étaient de véritables conflits qui nous opposaient, lui en tant qu'auteur, moi en tant que metteur en scène.

6 Pour commencer, qui allait jouer le rôle du farfûr? ... Youssef Idris était fanatique du grand artiste - aujourd'hui disparu - 'Abd al-Mun'im Ibrâhîm, alors qu'à mon avis, il fallait trouver un acteur peut-être pas aussi célèbre, mais à la silhouette menue, suscitant la sympathie. Mon choix se fixa sur 'Abd al-Salâm Muhammad... Il n'avait pas alors l'envergure de 'Abd al-Mun'im Ibrâhîm, et c'est à regret que Youssef Idris se rangea à mon avis. Je me mis à travailler le texte en concentrant mon attention d'abord sur le contenu, sans toutefois négliger l'autre aspect, celui de la forme scénique, du mode de représentation populaire préconisé par l'auteur.

7 Honnêtement, je dois dire que ce qui m'avait attiré dans le texte, c'était sa manière d'affronter la question de la démocratie avec un courage qui confinait à la témérité, bravant les valeurs en cours, engageant un duel chevaleresque avec l'interdit qui frappait alors le libre débat dans certains domaines; un duel opposant gouvernant et gouverné dans le cadre d'une dialectique burlesque dont la profondeur était à la mesure des blessures du passé et reflétait la vision d'un penseur s'efforçant de théoriser cet affrontement, de le concrétiser par une forme populaire de représentation dramatique.

8 Al-farâfir présentait donc deux aspects; le premier concernait la forme nouvelle que devait impliquer un théâtre nouveau puisant dans le répertoire et dans le matériel expressif du terroir égyptien; le second et le plus dangereux, se rapportait au débat intellectuel implicitement présent dans le contenu dramatique, et que d'autres s'étaient bien gardés d'approcher tant cela équivalait, à l'époque, à se jeter dans le brasier auquel s'étaient brûlés nombre de penseurs et d'écrivains des années 60 .

Mon enthousiasme pour le contenu de la pièce était lié à ma façon de voir les choses, laquelle n'a pas changé... Pour moi, l'engagement de l'artiste dans la réalité qui l'entoure, dans la société au sein de laquelle il vit, auprès de l'homme de son temps, devait nécessairement se concrétiser dans son rapport dialectique - perçu comme un droit, une responsabilité - avec celui dont il subit l'autorité. Le mot d'ordre de théâtre politique, ou des rapports entre théâtre et politique, se proposait comme sujet de controverse... Articles et conférences, approbations et critiques se manifestèrent dans divers endroits dont, entre autres, «Le Théâtre de Poche » que je dirigeais à l'époque. Pour ma part, je pensais et pense toujours que théatre et politique participent d'une même entité et que la pratique de l'art, dans sa plus simple expression, constitue déjà une sorte de choix politique, d'orientation, déterminé par une philosophie qui se concrétise à travers des formes esthétiques sélectionnées avec le plus grand soin, visant un but précis ayant des dimensions sociales; et quelques soient les formes d'expression mises en oeuvre dans la pratique de l'art, celle-ci finit par verser dans l'action » politique. 
10 point de vue formel, la pièce péchait par l'absence d'événement, d'intrigue délimitant les itinéraires divergents des personnages et qu'ainsi elle dérogeait aux règles que nous avaient apprises nos études académiques... Cependant, cette lacune elle-même agissait comme un stimulant pour l'imagination scénique... Je devais inventer des formes nouvelles, évoluées, pour théâtraliser l'antagonisme chronique opposant le farfûr à son maître, malgré l'absence de l'intrigue traditionnelle exigée par une situation dramatique. C'est ce qui m'a probablement poussé à travailler le texte en me plaçant dans la logique de l'auteur, c'est-à-dire en partant d'une perspective dialectique. Tantôt je procédai à la désintégration d'un des personnages en plusieurs unités avec lesquelles je constituais un choeur, ce qui m'a également amené à faire du public, quoique formellement, un des protagonistes de l'action scénique, par l'élimination de ce qu'on appelle « le quatrième mur », le parterre et la scène ne faisant plus qu'un... Tantôt je donnai au valet le rôle d'un revendeur et il allait dans la salle, proposant sa marchandise d'idées et de valeurs qu'il appelait «objets d'occasion »... Pour finir, le farfûr devait exécuter un mouvement circulaire dont le centre, le foyer était le maître ; la trajectoire s'élargissait progressivement jusqu'à englober l'amphithéâtre et le public... J'ai dû également me défaire de l'outillage scénique traditionnel comme le trou du souffleur, le rideau, les feux de la rampe et autres détails. Nous avons ainsi abouti à créer une arène, une sorte de théâtre dans le théâtre.

11 Il était dès lors évident qu'un conflit devait surgir entre, d'une part, la création dramatique ou mise en scène, située dans un présent temporel et, d'autre part, la création littéraire, le texte dramatique qui précède la mise en scène et se situe donc dans un passé temporel. Il était de même tout à fait normal que surgisse le problème de la marge de liberté d'interprétation permise au metteur en scène de sorte qu'il ne soit pas porté atteinte aux droits de l'auteur sur son texte.

Cette question a été exprimée par la formule du «metteur en scène créateur de la représentation dramatique "... Ce qui est étrange, c'est qu'elle avait déjà été résolue, et depuis des années, par le théâtre européen, alors que chez nous, dans les années 60 , elle servait encore de combustible propre à nourrir les litiges entre l'auteur, défendu par tout porteur de plume, et le metteur en scène que j'étais, qui avait osé proclamer que l'interprète d'un texte littéraire était maître de la vision créatrice qui se matérialisait dans la représentation dramatique. Je m'appuyais là sur un argument non équivoque, à savoir que le texte appartient à son auteur tant qu'il reste serré dans la reliure du livre, mais que tout lecteur/récepteur de ce texte - et pas nécessairement le metteur en scène - en devient aussi le créateur par l'action de son imagination.

En outre, si la représentation dramatique a pour base un support littéraire qui est le texte, elle a pour essence la moisson de toutes les créations et connaissances humaines, des expériences intimes, des éléments originaux, formels ou expressifs, auditifs ou visuels qui, en définitive, transforment le produit premier de l'art : ce dernier trouve alors sa place dans une nouvelle catégorie où la forme expressive relève du réalisateur. Il ne peut s'agir en aucune manière d'empiétement sur la propriété de l'auteur dans la mesure où, seule la création littéraire le concerne. Reste à espérer que l'on se souviendra de cette querelle - dont les échos se répercutèrent avec une ampleur considérable en beaucoup de tribunes - comme d'une contribution positive à la ferveur culturelle, critique et artistique qui caractérisait l'époque.

Égypte/Monde arabe, 7 | 1991 
14 Si la bataille a été chaude, ses acquis sur le plan du dialogue démocratique et la générosité avec lequel ce dernier fut mené, ont témoigné tant de la qualité morale du débat que de la supériorité de ceux dont les choix avaient commandé le fond et la forme. Autant de probité dans le comportement nous fait regretter l'absence de telles batailles menées avec de telles armes... Nous nous trouvons aujourd'hui aux prises avec des mesquineries qui occupent un espace considérable dans notre presse, sans autre objectif que de porter atteinte aux personnes, aux valeurs, à la morale et même à l'honneur d'autrui...

15 A la vérité, Youssef Idris défendait une double cause : d'une part, il affirmait son droit sur son texte et son refus de considérer la représentation dramatique comme une oeuvre de création en soi, relevant du metteur en scène. D'autre part, il défendait bec et ongles des thèses préconisant la quête de formes nouvelles, populaires, tirées du terroir égyptien, et réfutait toute formule aristotélicienne ou traditionnelle venue de l'Occident. Il s'est efforcé, dans une série d'articles et d'essais sur le théâtre et la littérature, de mettre au point sa théorie de la «théâtralisation de soi $»^{1}$, appelant à plonger ses racines dans le patrimoine populaire, le folklore, la tradition orale et notamment dans celle du théâtre d'ombres et de Karagheuz ${ }^{2}$ représentés sur les aires des villages où l'on bat le grain... On ne peut que louer un tel effort théorique, même si tout au long de ses années de création littéraire ultérieures à 1964, Youssef Idris n'a pas concrétisé cet effort sous une forme artistique authentique et achevée.

Il me reste à dire que l'expérience de la mise en scène d'Al-farâfir, malgré l'accueil positif que lui avait réservé la critique et qui aurait pu mettre l'eau à la bouche de certains - m'avait placé devant un défi : il tenait à ma conviction profonde que le sens des manifestations et célébrations populaires relève moins du contenu que de la forme et que, partant, il était possible d'aborder les classiques avec des formes d'expression et d'art populaires à travers lesquelles nous pouvons nous reconnaître, tout en éliminant l'obstacle dû à des contenus dépendants d'un mode de pensée ou d'un lieu géographique qui pourraient nous être étrangers. C'est cela qui a probablement déterminé mon choix, conscient ou non, des formes esthétiques dans maintes expériences menées après Al-farâfir. Je me contenterai ici d'évoquer les deux expériences qui ont immédiatement suivi, toujours en 1964 ; il s'agit de Yerma de Garcia Lorca, et de Yasin et Bahia de Nagib Surûr. J'ai traité le texte espagnol de "Yerma » cette héroïne symbolisant la stérilité, la privation, la soif, et qui figurait l'Espagne à une certaine époque - de manière à lui faire exprimer la virginité du sol égyptien, son attente de la graine, de l'eau et de la musique des pâtres. Le contenu ne m'avait pas dépaysé et l'expression esthétique que je lui ai donnée foisonnait de formes folkloriques et populaires que l'on retrouve dans les célébrations d'anniversaire, de mariage, en particulier à la campagne et en Haute-Égypte.

Il s'agissait du même contenu - mais avec une prédominance narrative - dans l'oeuvre de Nagib Surûr reprenant le fameux conte de la tradition orale populaire. Mon approche de la "poésie » fut donc la même que pour Al-farâfîr : c'étaient, chez Surûr, des personnages abstraits, relevant du récit et qu'il fallait matérialiser, personnifier sur la scène, de manière à créer un contraste entre le récit du narrateur et le vécu du personnage lui-même. Il y eut donc, d'une part, le ou les narrateurs exprimant la voix du poète, et de l'autre, l'événement concrétisé par le «fait» poétique incarné Yassine, Bahia, le père, la mère ou l'oncle... - sur la scène où se déroulait le récit matérialisé du drame, dans un cadre évoquant les festivités qui président aux veillées 
du Ramadan dans les cours ou sur les places des villages de Haute-Egypte, et que nous appelons parfois sâmir.

Ces deux manières d'aborder Lorca et Nagib Surûr - (avec Va Bahia wa khabbirîni $i^{3}$, le second a persisté dans la voie de la tradition populaire et des formes expressives inspirées du terroir) - s'étaient imposées à moi en tant que metteur en scène, dès mes débuts avec Al-farâfir, lorsque je me représentais la dialectique du rapport entre le temps de l'écriture, appartenant au passé, et le temps présent, celui du spectateur, ou en d'autres termes, le temps où coïncidaient le champ de la création et le champ de la réceptivité. Ces approches s'étaient donc efforcées d'assimiler les caractéristiques des arts populaires d'improvisation, qui relèvent toutes du « langage expressif $»^{4}$. Quant au contenu, même s'il devait appartenir au patrimoine grec et qu'il se fût agi d'Agamemnon, il n'y aurait pas eu d'obstacle à sa compréhension s'il était interprété et exprimé avec les formes, les costumes, la musique ou les gestes inspirés de notre milieu.

19 Si j'ai évoqué ces exemples, c'est surtout pour bien souligner l'importance de l'appel de Youssef Idris - invitant à effectuer un retour conscient aux origines et à s'insurger contre les assauts culturels et artistiques venus de l'étranger - lequel avait provoqué un débat et avait eu un tel retentissement qu'il avait rallié de nombreux écrivains, auteurs arabes d'Egypte ou d'ailleurs, qui s'étaient armés des techniques dramatiques qu'il préconisait... Autant de tentatives, d'effort sérieux et sincères pour la plupart, qui cherchaient à réaliser son voeu.

Telles sont les réflexions que m'ont inspiré mes feuillets de souvenirs où Youssef Idris occupe une grande place, en tant que créateur, combattant audacieux et en tant qu'ami, un ami que j'ai d'autant plus aimé que je le connaissais mieux... Ce qui, en outre, m'a poussé à évoquer ces souvenirs, c'est la rare originalité de cet auteur, dont on n'apprécie peut-être pas la valeur aujourd'hui, en se repliant définitivement vers l'époque où ses oeuvres furent considérées comme unassaut de l'inconnu, comme une insurrection quasi-suicidaire contre des formes sclérosées que beaucoup hésitaient pourtant à remettre en cause.

21 Youssef Idris fut un exemple de courage et de ferveur lorsqu'il prit parti pour la spontanéité dans l'écriture de la nouvelle, où il occupa le premier rang, dans récriture dramatique et enfin dans la chronique quotidienne où il reflétait le sentiment de l'homme de la rue avec cette sincérité, cet enthousiasme profonds que nous souhaitons en vain trouver chez bien des chroniqueurs. Youssef Idris s'était aussi fait, par le biais des médias, l'avocat de causes humaines locales ou mondiales. Que Youssef Idris ait été tout cela nous fait sentir d'autant plus cruellement sa perte, sa fiévreuse impulsivité nous manque tout comme son grand rêve d'un théâtre dont il voyait, sans doute plus que d'autres, se dessiner les principales caractéristiques, et son rêve noble d'un citoyen libéré. Il nous reste à relire avec lucidité ses oeuvres diverses; nous y découvrirons sûrement des trésors que lui-même n'avait probablement pas soupçonnés, et que, le plus souvent, nous n'avons pas estimés à leur juste valeur. 


\section{NOTES}

1. "Théâtralisation de soi »: ou configuration dramatique de soi.

2. Karagheuz (en Egypte : karakoch, ou Ara'och en langue dialectale ; en Syrie, etc... : karakoch): mot d'origine turque. C'est le nom du personnage principal qui anime le théâtre d'ombres des pays méditerranéens. Ses farces et sa verve sont inépuisables. Les variantes de ce personnage constantinoplois se sont répandues en Egypte, Syrie, etc... ainsi que dans les pays du Maghreb.

3. Yâ Bahia wâ Khabbirini : «O Bahia... Raconte moi »

4. "Langage expressif » : ou forme expressive, pour différencier d'avec la langue parlée. Le mot « langage » est utilisé ici dans le sens de système ou ensemble de signes n'appartenant pas à la langue et qui sont - utilisés par unités séparées ou combinées - porteurs d'un message.

INDEX

Mots-clés : Idris (Youssef), littérature 